\title{
ON THE HOLONOMY GROUP OF THE CONFORMALLY FLAT RIEMANNIAN MANIFOLD
}

\author{
MINORU KURITA
}

The main purpose of the present paper is to show that the local homogeneous holonomy group of the conformally flat Riemannian manifold is the full rotation group with some exceptions.

1. Let $M$ be an $n$-dimensional conformally flat Riemannian manifold $\left(n \geqslant 3\right.$ ), the metric being given by $d s^{2}=a^{2} \sum d x_{i}^{2}$ in a coordinates neighborhood $U$ with a function $a=a\left(x_{1}, \ldots, x_{n}\right)$ of class 2 . We take rectangular frames in the tangent spaces at each point of $U$ and put according to the frames

$$
d s^{2}=\sum \omega_{i}^{2}
$$

When we put

$$
\omega_{i}=a \pi_{i},
$$

$\sum \pi_{i}$ is a flat metric, and if we take $\pi_{i j}$ such that

$$
d \pi_{i}=\pi_{j \wedge} \pi_{j i}, \quad \pi_{i j}=-\pi j i,
$$

then we have by the flatness of $\sum \pi_{i}^{?}$

$$
d \pi_{i j}=\pi_{i k \wedge} \pi_{k j}
$$

Next we put

$$
\begin{gathered}
d a / a=b_{i} \pi_{i} \\
\omega_{i j}=\pi_{i j}+b_{i} \pi_{j}-b_{j} \pi_{i}
\end{gathered}
$$

and we get

$$
d \omega_{i}=\omega_{j \wedge} \omega_{j i}, \quad \omega_{i j}=-\omega_{j i} .
$$

Thus $\omega_{i j}$ are the parameters of the Riemannian connection of $M$ in $U$. Now we calculate the curvature forms of $M$. Putting

Received June 10, 1955. 


$$
\begin{gathered}
\sum b_{i}^{2}=b^{2} \\
d b_{i}+b_{k} \omega_{k i}-\frac{1}{2} b^{2} \pi_{i}=a^{2} \lambda_{i k} \pi_{k}
\end{gathered}
$$

we get for the curvature forms

$$
\Omega_{i j}=d \omega_{i j}-\omega_{i k \wedge} \omega_{k j}=\lambda_{i k} \omega_{k} \wedge \omega_{j}+\omega_{i} \wedge \lambda_{j k} \omega_{k},
$$

using the relations (3), (4), (6). Taking an exterior differential of (5) we get

$$
\lambda_{i j}=\lambda_{j i}
$$

We put $\Omega_{i j}=\frac{1}{2} R_{i j k h} \omega_{k} \wedge \omega_{h}\left(R_{i j k h}=-R_{i j h k}\right)$ and we have by $(10)$

$$
R_{i j k h}=\lambda_{i k} \delta_{j h}-\lambda_{i h} \delta_{j k}-\lambda_{j k} \delta_{i h}+\lambda_{j h} \delta_{i k} .
$$

Contracting with respect to $j$ and $h$ we get Ricci's tensor

$$
R_{i k}=(n-2) \lambda_{i k}+\lambda_{j j}, \quad R=R_{i i}=2(n-1) \lambda_{j j} .
$$

Hence we can represent $\lambda_{i j}$ as follows,

$$
\lambda_{i j}=\frac{1}{n-2}\left(R_{i j}-\frac{1}{2(n-1)} R \delta_{i j}\right) .
$$

We take a geodesic of the manifold $M$. It satisfies the differental equations

$$
\frac{d}{d s}\left(\frac{\omega_{i}}{d s}\right)+\frac{\omega_{j}}{d s} \frac{\omega_{j i}}{d s}=0
$$

and along it we have by (5), (9)

$$
\frac{\lambda_{i j} \omega_{i} \omega_{j}}{d s^{2}}=\frac{d}{d s}\left(\frac{1}{a} \frac{d a}{d s}\right)+\left(\frac{1}{a} \frac{d a}{d s}\right)^{2}-\frac{1}{2} \frac{b^{2}}{a^{2}} .
$$

This gives an interpretation of the tensor $\left(\lambda_{i j}\right)$ in terms of $a$ and $b$.

2. We assume that our metric is not flat in the coordinate neighborhood $U$ and the local homogeneous holonomy group $H_{p}$ (cf. [1]) at any point of $U$ is not the full rotation group $S O(n)$. Then we take a point $p$ at which the tensor $\left(\lambda_{i j}\right)$ is not a zero tensor. When we take a suitable rectangular frame $\mathbf{e}_{1}, \ldots, \mathbf{e}_{n}$ at $p$, we can reduce the tensor $\left(\lambda_{i j}\right)$ into the diagonal form with the diagonal elements $\lambda_{1}, \ldots, \lambda_{n}$. Then we get for $(10)$

$$
\left.\Omega_{i j}=\left(\lambda_{i}+\lambda_{j}\right) \omega_{i} \wedge \omega_{j} \quad \text { (not summed for } i, j\right)
$$

at $\not$. Conversely if the curvature forms are represented as (15) by a suitable choice of rectangular frames, the manifold is conformally flat under the as- 
sumption $n \geqslant 4$. This can be verified by the vanishing of the conformal curvature tensor.

We denote by $H_{p}$ the local homogeneous holonomy group at $p$ and by $\mathscr{S}_{p}$ the Lie algebra of $H_{p}$. Let $S_{i j}$ be a skew symmetric matrix $\left(s_{h l}\right)$ such that $s_{i j}=-s_{j i}=1$ and all the other $s_{h l}$ 's are zero. Then by virtue of (15) $\mathfrak{S}_{p}$ contains a subalgebra generated by $S_{i j}$ if $\lambda_{i}+\lambda_{j} \neq 0$. Let $O\left(i_{1}, \ldots, i_{k}\right)$ be a subgroup of a full rotation group $S O(n)$ which induces the full rotation in the linear subspaces generated by $\mathbf{e}_{i_{1}}, \ldots, \mathbf{e}_{i_{k}}$ and fixes the remaining fundamental vectors. As at least one of $\lambda_{i}+\lambda_{j}$ is not zero at $p, \mathscr{S}_{p}$ contains $S_{i j}$ for such $i$ and $j$. Hence $H_{p}$ contains $O(i, j)$. If $H_{p}$ is not the full rotation group, there exist $k$ and $i_{1}, \ldots, i_{k}$ such that $H_{p}$ contains $O\left(i_{1}, \ldots, i_{k}\right)$ but does not contain $O\left(i_{1}, \ldots, i_{k}, j\right)$ for any $j$. We change the indices suitably and assume that $H_{p}$ contains $O(1, \ldots, k)$ but does not contain $O(1, \ldots, k, j)$ for any $j$. Then $\mathfrak{H}_{p}$ does not contain $S_{a r}(a=1, \ldots, k ; r=k+1, \ldots, n)$ and we get

$$
\lambda_{a}+\lambda_{r}=0 \quad(a=1, \ldots, k ; r=k+1, \ldots, n) .
$$

By putting $\lambda=\lambda_{1}$ we have

$$
\lambda_{1}=\ldots=\lambda_{k}=\lambda, \quad \lambda_{k+1}=\ldots=\lambda_{n}=-\lambda \quad(\lambda \neq 0) .
$$

In this section and the next we assume that the indices run as follows,

$$
a, b=1, \ldots, k \quad r, s=k+1, \ldots, n \quad i, j=1, \ldots, n .
$$

We have by (15)

$$
\Omega_{a b}=2 \lambda \omega_{a \wedge} \omega b, \quad \Omega_{r s}=-2 \lambda \omega_{r} \wedge \omega_{s} \text { all the others zero. }
$$

Next we take a point $q$ in the neighborhood of $p$ and choose a suitable rectangular frame at the point. Then we have at $q$

$$
\left.\Omega_{i j}=\left(\lambda_{i}+\lambda_{j}\right) \omega_{i \wedge} \omega_{j} \quad \text { (not summed for } i, j\right) .
$$

The eigenvalues of the symmetric matrix $\left(\lambda_{i j}\right)$ are continuous with respect to the values of $\lambda_{i j}$ in the sense that those of $\left(\lambda_{i j}\right)$ for $\lambda_{i j}=a_{i j}$ are in arbitrary small neighborhoods of those for $\lambda_{i j}=b_{i j}$ if $a_{i j}$ 's are sufficiently near to $b_{i j}$. When the point $q$ is sufficiently near to $p$, we conclude by (17)

$$
\Omega_{a b}=\left(\lambda_{a}+\lambda_{b}\right) \omega_{a \wedge} \omega_{b} \neq 0, \quad \Omega_{r s}=\left(\lambda_{r}+\lambda_{s}\right) \omega_{r} \wedge \omega_{s} \neq 0
$$

(not summed for $a, b, r, s$ ) 
as it is so at the point $p$. Hence $H_{q}$ contains $S_{a b}$ and $S_{r s}$ and so $H_{q}$ contains $O(1, \ldots, k)$ and $O(k+1, \ldots, n)$. If $H_{q}$ contains none of $O(1, \ldots, k, j)$, (17) holds good at $q$. If $H_{q}$ contains at least one of $O(1, \ldots, k, j)$, we change the indices of $\mathbf{e}_{k+1}, \ldots, \mathbf{e}_{n}$ in such a way that $H_{q}$ contains $O(1, \ldots, k, k+1$, $\ldots, k+l)$ and none of $O(1, \ldots, k, k+1, \ldots, k+l, k+l+m)$. Then we get by the repetition of the above process

$$
\Omega_{r s}=0 \quad(r=k+1, \ldots, k+l ; s=k+l+1, \ldots, n) .
$$

This contradicts (18). Thus we can take frames at each point of $U$ in such a way that (17) holds good with the same $k$. These frames can be so taken as to satisfy the differentiability, because the eigenvalues of $\left(\lambda_{i j}\right)$ satisfy the relation (16) and the process of transforming $\left(\lambda_{i j}\right)$ into the diagonal form can be taken analytic except at the point such that $\lambda_{1}=\ldots=\lambda_{n}$, namely $\lambda=0$. These circumstances are discussed precisely in section 7 . We treat at first the neighborhood in which $\lambda$ never vanishes, the non-existence of the point at which $\lambda=0$ being assured thereafter.

3. The next step is to find the Riemannian metric which satisfies the relation (17). In this sectin we treat the case $n-k \geqslant 2, k \geqslant 2$.

By Bianchi's identity we have

$$
d \Omega_{a r}=-\Omega_{a i} \wedge \omega_{i r}+\omega_{a i} \wedge \Omega_{i r} .
$$

By virtue of (17) we get

$$
0=-\Omega_{a b \wedge} \omega_{b r}+\omega_{a s \wedge} \Omega_{s r}=-2 \lambda\left(\omega_{a \wedge} \omega_{b \wedge} \omega_{b r}+\omega_{a s \wedge} \omega_{s \wedge} \omega_{r}\right) .
$$

Putting $\omega_{a s}=A_{a s i} \omega_{i}$ we get by the assumption $\lambda \neq 0$

$$
\omega_{a \wedge} \omega_{b \wedge} A_{b r i} \omega_{i}+A_{a s i} \omega_{i} \wedge \omega_{s \wedge} \omega_{r}=0 .
$$

As $n-k \geqslant 2, k \geqslant 2$, we have

$$
A_{b r s}=0, \quad A_{a s b}=0
$$

and so

$$
\omega_{a r}=-\omega_{r a}=0
$$

Consequently (7) takes the forms

$$
d \omega_{a}=\omega_{i} \wedge \omega_{i a}=\omega_{b \wedge} \omega_{b a}, \quad d \omega_{r}=\omega_{i \wedge} \omega_{i r}=\omega_{s \wedge} \omega_{s r}
$$

and by E. Cartan's lemma the metric of our manifold decomposes, namely 


$$
d s^{2}=d s_{1}^{2}+d s_{2}^{2}
$$

where

$$
d s_{1}^{2}=g_{a b}\left(x_{1}, \ldots, x_{k}\right) d x_{a} d x_{b}, \quad d s_{2}^{2}=g_{r s}\left(x_{k+1}, \ldots, x_{n}\right) d x_{r} d x_{s}
$$

with suitably chosen coordinates. When we take suitably chosen rectangular frames in each manifold with the metric $d s_{1}^{2}$ and $d s_{2}^{2}$, $\omega_{a}$ 's are expressed by the coordinates $x_{1}, \ldots, x_{k}$ and $\omega_{r}$ 's by $x_{k+1}, \ldots, x_{n}$. As the relations (17) holds good, $\lambda$ is constant and $d s_{1}^{2}$ is a metric of constant curvature $K$, while $d s_{2}^{2}$ is one of constant curvature $-K$.

Thus any point of $U$ at which $\lambda$ does not vanish has a neighborhood in which (16) holds good with non zero constant. The set $V$ of all points at which (16) holds good with the same constant is open. On the other hand $V$ is closed as $\lambda_{i}$ 's are continuous. $\quad V$ is closed and open in $U$. As $U$ is connected, $V$ coincides with $U$ and there is no point in $U$ at which $\lambda=0$.

4. Next we treat the case $n-k=1, n \geqslant 4$. In this section we assume that the indices run as $a, b, c=1, \ldots, n-1$. By (17)

$$
\Omega_{a b}=2 \lambda \omega_{a} \wedge \omega_{b}, \quad \Omega_{a n}=0 .
$$

By virtue of (19) we get $A_{a n n}=0$ and we can put

$$
\omega_{a n}=A_{a b} \omega b \text {. }
$$

By (21) we have for Bianchi's identity

$$
d \Omega_{a b}=-\Omega_{a c} \wedge \omega_{c b}+\omega_{a c} \wedge \Omega_{c b}
$$

and this can be written as

$$
d \lambda \wedge \omega_{a} \wedge \omega_{b}+\lambda d \omega_{a} \wedge \omega_{b}-\lambda \omega_{a} \wedge d \omega_{b}=-\lambda \omega_{a} \wedge \omega_{c} \wedge \omega_{c b}+\omega_{a c} \wedge \lambda \omega_{c} \wedge \omega_{b} .
$$

Hence by (7) and (21)

$$
d \lambda \wedge \omega_{a} \wedge \omega_{b}=\lambda \omega_{n \wedge}\left(A_{b c} \omega_{a} \wedge \omega_{c}+A_{a c} \omega_{c} \wedge \omega_{b}\right)
$$

and so

$$
A_{b c}=0 \quad(c \neq a, b) .
$$

Putting $A_{a}=A_{a a}$ (not summed for $a$ ) we get

$$
d \lambda-\lambda\left(A_{a}+A_{b}\right) \omega_{n}=p \omega_{a}+q \omega_{b}
$$

for all $a$ and $b(a \neq b)$. As $k \geqslant 3, A_{a}+A_{b}$ is independent of $a$ and $b$ and so $A_{a}$ 
is independent of $a$. We put $A_{a}=A$. We also get from (23) $p=0, q=0$. Thus we have

$$
d \lambda-2 \lambda A \omega_{n}=0, \quad \omega_{a n}=A \omega_{a} .
$$

By the relation (17) we get

$$
\begin{aligned}
0 & =\Omega_{a n}=d \omega_{a n}-\omega_{a b} \wedge \omega_{b n}=d A \wedge \omega_{a}+A d \omega_{a}-\omega_{a b} \wedge A \omega_{b} \\
& =d A_{\wedge} \omega_{a}+A \omega_{n} \wedge \omega_{n a}=\left(d A-A^{2} \omega_{n}\right) \wedge \omega_{a} .
\end{aligned}
$$

Hence

$$
d A=A^{2} \omega_{n}
$$

In the neighborhood of the point at which $A \neq 0$ we have

$$
d A / A^{2}=\omega_{n} .
$$

Hence putting $x_{n}=-A^{-1}$ we get

$$
\omega_{n}=d x_{n}
$$

and by virtue of $(24)$

$$
d \lambda=-2 \lambda / x_{n} \cdot d x_{n}, \quad \lambda=C / x_{n}^{2}(C \text { const })
$$

and

$$
\omega_{a n}=-\omega_{a} / x_{n}
$$

Hence

$$
d \omega_{a}=\omega_{b \wedge} \omega_{b a}+\omega_{n \wedge} \omega_{n a}=\omega_{b \wedge} \omega_{b a}+d x_{n \wedge} \omega_{a} / x_{n} .
$$

Putting $\omega_{a}=x_{n} \rho_{a}$ we get

$$
d \rho_{a}=\rho_{b} \wedge \omega_{b a}
$$

and

$$
d \sigma^{2}=\sum \rho_{a}^{2}=g_{a b}\left(x_{1}, \ldots, x_{n-1}\right) d x_{a} d x_{b}
$$

for suitably chosen coordinates $x_{1}, \ldots, x_{n-1}$. Also we have

$$
\begin{aligned}
d \omega_{a b} & -\omega_{a c} \wedge \omega_{c b}=\Omega_{a b}+\omega_{a n} \wedge \omega_{n b}=2 \lambda \omega_{a \wedge} \omega_{b}-A^{2} \omega_{a \wedge} \omega_{b} \\
& =\left(2 \lambda x_{n}^{2}-A^{2} x_{n}^{2}\right) \rho_{a} \wedge \rho_{b}=(2 C-1) \rho_{a} \wedge \rho_{b} .
\end{aligned}
$$

Hence $d \sigma^{2}$ is a metric of constant curvature, and our metric is

$$
d s^{2}=x_{n}^{2} d \sigma^{2}+d x_{n}^{2} .
$$

But the local homogeneous holonomy group $H_{p}$ of this manifold does not keep the direction $\mathbf{e}_{n}$ invariant. As the holonomy group $H_{p}$ contains $O(1, \ldots, n-1)$, $H_{p}$ is a full rotation group. The manifold affords an example of the one for 
which the curvature forms satisfy the relations (21) for suitably chosen frames and yet the metric does not decompose.

Now we need only to treat the case that $A$ is identically zero. Then by virtue of (24) $\lambda$ is constant and $\omega_{a n}=0$ and we get

$$
d s^{2}=d \sigma^{2}+d x_{n}^{2}
$$

where $d \sigma^{2}$ is a metric of constant curvature. This holds good in $U$ by the same discussion as at the end of the preceding section.

5. Lastly we treat the case $n=3$. If the group $H_{p}$ is not the full rotation group in $U$, it is reducible and we can take rectangular frames in such a way that the metric can be written as

$$
d s^{2}=\omega_{1}^{2}+\omega_{2}^{2}+\omega_{3}^{2}
$$

where $\omega_{1}, \omega_{2}$ are Pfaffian forms in the variables $x_{1}, x_{2}$ and

$$
\omega_{3}=d x_{3} .
$$

For the parameters $\omega_{i j}$ of the Riemannian connection we have

$$
\omega_{13}=0, \quad \omega_{23}=0,
$$

and for curvature forms

$$
\Omega_{12}=-K \omega_{1 \wedge} \omega_{2}, \quad \Omega_{13}=0, \quad \Omega_{23}=0 .
$$

For Ricci's tensor we have

$$
R_{11}=-K, \quad R_{22}=-K, \text { all the others zero } R=-2 K .
$$

Now a 3-dimensional Riemannian manifold with the metric $d s^{2}=\sum \omega_{i}^{2}$ is conformally flat when and only when

$$
D p_{i k \wedge} \omega_{k}=0,
$$

where $\boldsymbol{D}$ denotes covariant differential and

$$
p_{i j}=-\frac{1}{n-2}\left(R_{i j}-\frac{1}{2(n-1)} R \delta_{i j}\right) .
$$

This is a formulation in rectangular frames of the well known property. In our case $p_{i j}$ reduces to

$$
p_{i j}=-R_{i j}+\frac{1}{4} R \delta_{i j}
$$


and we have by (29)

$$
p_{11}=\frac{1}{2} K, \quad p_{22}=\frac{1}{2} K \quad \text { all the other } p_{i j} \text { vanish. }
$$

By these and (28) the relation (30) reduces to $d K_{\wedge} \omega_{1}=0, d K_{\wedge} \omega_{2}=0$ and so $d K=0$ and $K$ is constant. Hence our metric (26) is conformally flat when and only when $d \sigma^{2}=\omega_{1}^{2}+\omega_{2}^{2}$ is of constant curvature. Thus the metric for which (30) holds good in some neighborhood has been found.

6. In summary we get the following theorem.

THEOREM. The local homogeneous holonomy group $H_{p}$ of an n-dimensional conformally flat Riemannian manifold of class $C_{2}$ is in general the full rotation group $S O(n)$. If $H_{p}$ is not $S O(n)$ for any point of a coordinate neighborhood $U$, we can classify into the following three cases:

1) $H_{p}$ is an identity and the metric is flat in $U$.

2) $H_{p}$ is $S O(k) \times S O(n-k)$ and $U$ is a direct product of a k-dimensional manifold of constant curvature $K$ and an $n-k$-dimensional manifold of constant curvatur $-K(K \neq 0)$.

3) $H_{p}$ is $S O(n-1)$ and $U$ is a direct product of a straight line (or a segment) and an n-1-dimensional manifold of constant curvature.

The set $N$ of all the point of $M$ at which $H_{p}$ is $S O(n)$ is closed. In fact when $p$ is a limit point of $N$ any neighborhood of $p$ has a point $q \in N$ and by Lemma 3.2 in [1] we have $H_{p} \supset H_{q}=S O(n)$ and so $H_{p}=S O(n)$. For any point $p$ at which $H_{p} \neq S O(n)$ (if exist) we can take a neighborhood $U$ such that $U_{\frown} N$ is empty and by our theorem one of 1),2),3) holds good in $U$. By the Theorem 3 in [1] we can conclude that if there is no point on an $n$-dimensional conformally flat connected manifold $M$ at which $H_{p}$ is $S O(n)$, the restricted homogeneous holonomy group of $M$ is an identity, or $S O(k) \times S O(n-k)$, or $S O(n-1) . \quad$ In fact $\lambda_{i}$ 's in our discussion are continuous and (17) holds good for all the points of $M$ with the same number $k$ and $\operatorname{dim} H_{p}$ is constant over $M$. An example of the case in which $H_{p}$ is $S O(n)$ in some points and $S O(k)$ $\times S O(n-k)$ in other points can easily be given.

7. Here we give an attention to a symmetric covariant tensor field of second order over an $n$-dimensional Riemannian manifold. Let the components 
of the tensor with respect to certain rectangular frames be $\left(a_{i j}\right)$. When the metric, frames and $\left(a_{i j}\right)$ satisfy certain differentiability conditions (for example class $\left.C_{k}\right)$ it is not sure that the frames can be taken in such a way that $\left(a_{i j}\right)$ reduces to a diagonal form and yet the frames satisfy the differentiability of the same kind. An example is given by a symmetric tensor field with the components

$$
\begin{gathered}
a_{11}=p_{1} \cos ^{2} \theta+p_{2} \sin ^{2} \theta, \quad a_{22}=p_{1} \sin ^{2} \theta+p_{2} \cos ^{2} \theta \\
a_{12}=a_{21}=2\left(p_{1}-p_{2}\right) \sin \theta \cos \theta,
\end{gathered}
$$

where $\theta=\frac{1}{x_{1}-x_{2}}, p_{1}-p_{2}=\left(x_{1}-x_{2}\right)^{n}\left(p_{i}\right.$ is class $\left.C_{n}\right)$, on the euclidean plane with the rectangular coordinates $x_{1}, x_{2}$. The eigenvalues of $\left(a_{i j}\right)$ are $p_{1}$ and $p_{2}$. But the angle of rotation which transforms the components of the tensor into a diagonal form is $\theta$ and it is not continuous at the points such that $x_{1}=x_{2}$. In general this singularity appears at the points at which the multiplicities of eigenvalues of $\left(a_{i j}\right)$ differ from those at the sufficiently near points. In fact if the multiplicities of the eigenvalues of $\left(a_{i j}\right)$ are each constant in some neighborhood, the eigenvalues are analytic functions of $\left(a_{i j}\right)$ as they are simple roots of the polynomials obtained from $\varphi(t)=\operatorname{det}\left(a_{i j}-\delta_{i j} t\right)$ by a suitable successive differentiation. When the eigenvalues are analytic functions of $\left(a_{i j}\right)$, we can transform it into a diagonal form by an analytic process. In the treatment of the preceding sections the relation (16) is satisfied and there was no obstacle to the discussion.

8. In a conformally flat Riemmanian manifold we can take rectangular frames such that the curvature forms reduce to

$$
\left.\Omega_{i j}=\left(\lambda_{i}+\lambda_{j}\right) \omega_{i \wedge} \omega_{j} \quad \text { (not summed for } i, j\right)
$$

and according to the discussion of the previous section this can be accomplished by a differentiable process with an exception of certain points. Analogously if we take suitable rectangular frames in an $n$-dimensional Riemannian manifold of imbedding class one, we have for the curvature forms

$$
\left.\Omega_{i j}=-k_{i} k_{j} \omega_{i \wedge} \omega_{j} \quad \text { (not summed for } i, j\right) .
$$

(31) is a sufficient condition for the conformal flatness if the dimension of the manifold is greater than 3 , while (32) is a sufficient condition for the imbedding class one if at least three of $k_{1}, \ldots, k_{n}$ are not zero. The latter is a different 
formulation of T. Y. Thomas' well known result and we can give a proof by using Bianchi's identities, the clue of the proof being the verification of the structural equations in the $n+1$-dimensional euclidean space.

Now we consider the Riemannian manifolds whose curvature forms reduce to

$$
\left.\Omega_{i j}=K_{i j} \omega_{i \wedge} \omega_{j} \quad \text { (not summed for } i, j\right)
$$

by a suitable choice of rectangular frames. We calculate Ricci's tensor and get

$$
R_{i j}=\delta_{i j} \sum_{k} K_{i k} \quad(\text { not summed for } i \text { ) }
$$

and so the fundamental vectors $\mathbf{e}_{1}, \ldots, \mathbf{e}_{n}$ are in Ricci's principal directions. As an application there exist in a conformally flat Riemannian manifold of imbedding class one rectangular frames for which curvature forms are represented at the same time by (31) and (32). The treatment in my previous paper [4] was along that line and the proofs of Theorem 3 in 2.2 and Theorem 4 in 2.3 could be simplified.

The manifolds whose curvature forms are represented by (33) for suitably chosen rectangular frames have some simple properties. If none of $K_{i j}$ is zero at a point $p$, the local homogeneous holonomy group $H_{p}$ at the point is $S O(n)$. For example $H_{p}$ of the Riemannian manifold of imbedding class one is $S O(n)$ if none of $k$ is zero. This is so for the closed hypersurfaces in the $n+1$ dimensional euclidean space, as was proved by S. Kobayasi [5]. If the group $H_{p}$ is $S O(n)$ the Riemannian manifold cannot be Kaehlerian, and hence the non-existence of non flat, conformally flat Kaehlerian manifold (cf. [2], p. 181) can be deduced, as well as the non-existence of the Kaehlerian manifold of imbedding class one such that none of $k_{i}$ is zero.

There exist for any Riemannian manifold closed differential forms such as

$$
\Omega_{i j \wedge} \Omega_{j i}, \quad \Omega_{i k \wedge} \Omega_{k j \wedge} \Omega_{j h \wedge} \Omega_{h i}, \ldots
$$

(cf. [3], p. 37). All these vanish for those manifolds which satisfy the relation (33). The geometrical characterization and examples of such manifolds except the conformally flat one and the one of imbedding class one are unknown to the author. 


\section{REFERENCES}

[1] A. Nijenhuis, On the holonomy groups of linear connections I a. General properties of affine connections, Indag. Math., 15, pp. 233-240 (1953).

[2] K. Yano and I. Mogi, On the real representation of Kaehlerian manifolds, Ann. of Math., 61, pp. 170-189 (1955).

[3] S. S. Chern, Topics in differential geometry, Princeton (1951).

[4] M. Kurita, On conformal Riemann spaces, Jour. Math. Soc. Japan, 7, pp. 13-31 (1955).

[5] S. Kobayasi, Holonomy groups of hypersurfaces, to appear in the next issue of Nagoya Math. Journ.

Mathematical Institute, Nagoya University 